\title{
The risk of mycobacterial infections associated with inhaled corticosteroid use
}

\author{
Sarah K. Brode 1,2,3, Michael A. Campitelli ${ }^{4}$, Jeffrey C. Kwong ${ }^{4,5,6,7}$, Hong Lu ${ }^{4}$, \\ Alex Marchand-Austin ${ }^{5}$, Andrea S. Gershon ${ }^{1,4,7,8}$, Frances B. Jamieson ${ }^{5,9}$ and \\ Theodore K. Marras ${ }^{1,2}$
}

Affiliations: ${ }^{1}$ Division of Respirology, Dept of Medicine, University of Toronto, Toronto, ON, Canada. ${ }^{2}$ Dept of Medicine, University Health Network and Mount Sinai Hospital, Toronto, ON, Canada. ${ }^{3}$ Division of Respiratory Medicine, West Park Healthcare Centre, Toronto, ON, Canada. ${ }^{4}$ Institute for Clinical Evaluative Sciences, Toronto, ON, Canada. ${ }^{5}$ Public Health Ontario, Toronto, ON, Canada. ${ }^{6}$ Dept of Family and Community Medicine, University of Toronto, Toronto, ON, Canada. ${ }^{7}$ Dalla Lana School of Public Health, University of Toronto, Toronto, ON, Canada. ${ }^{8}$ Dept of Medicine, Sunnybrook Health Sciences Centre, Toronto, ON, Canada. ${ }^{9}$ Dept of Laboratory Medicine and Pathobiology, University of Toronto, Toronto, ON, Canada.

Correspondence: Sarah K. Brode, Toronto Western Hospital, 7E-453, 399 Bathurst Street, Toronto, ON M5T 2S8, Canada. E-mail: sarah.brodeđuhn.ca

@ERSpublications

Inhaled corticosteroid use in older adults with obstructive lung disease increases with risk of NTM lung infection http://ow.ly/k2mL30cDO1b

Cite this article as: Brode SK, Campitelli MA, Kwong JC, et al. The risk of mycobacterial infections associated with inhaled corticosteroid use. Eur Respir J 2017; 50: 1700037 [https://doi.org/10.1183/ 13993003.00037-2017].

ABSTRACT Inhaled corticosteroid (ICS) use is associated with an increased risk of pneumonia. This study was performed to determine if ICS use is associated with an increased risk of nontuberculous mycobacterial pulmonary disease (NTM-PD) or tuberculosis (TB).

We conducted a population-based nested case-control study using linked laboratory and health administrative databases in Ontario, Canada, including adults aged $\geqslant 66$ years with treated obstructive lung disease (i.e. asthma, chronic obstructive pulmonary disease (COPD) or asthma-COPD overlap syndrome) between 2001 and 2013. We estimated odds ratios comparing ICS use with nonuse among NTM-PD and TB cases and controls using conditional logistic regression.

Among 417494 older adults with treated obstructive lung disease, we identified 2966 cases of NTM-PD and 327 cases of TB. Current ICS use was associated with NTM-PD compared with nonuse (adjusted OR (aOR) 1.86, 95\% CI 1.60-2.15) and was statistically significant for fluticasone (aOR 2.09, 95\% CI 1.80 2.43), but not for budesonide (aOR 1.19, 95\% CI 0.97-1.45). There was a strong dose-response relationship between incident NTM-PD and cumulative ICS dose over 1 year. There was no significant association between current ICS use and TB (aOR 1.43, 95\% CI 0.95-2.16).

This study suggests that ICS use is associated with an increased risk of NTM-PD, but not TB.

This article has supplementary material available from erj.ersjournals.com

Received: Jan 012017 | Accepted after revision: June 132017

Support statement: This study was supported by the Physician Services Inc. Foundation and by the Institute for Clinical Evaluative Sciences (ICES) and Public Health Ontario, which are funded by annual grants from the Ontario Ministry of Health and Long-Term Care (MOHLTC). The opinions, results and conclusions reported in this article are those of the authors and are independent from the funding sources. No endorsement by ICES or the Ontario MOHLTC is intended or should be inferred. Parts of this material are based on data and information compiled and provided by the Canadian Institute of Health Information (CIHI). However, the analyses, conclusions, opinions and statements expressed herein are those of the authors, and not necessarily those of CIHI. Funding information for this article has been deposited with the Crossref Funder Registry.

Conflict of interest: Disclosures can be found alongside this article at erj.ersjournals.com

Copyright @ERS 2017 


\section{Introduction}

Obstructive lung disease (OLD), comprising predominantly chronic obstructive pulmonary disease (COPD) and asthma, is very common. In Ontario, Canada, the lifetime risk of COPD and asthma is $28 \%$ and $35 \%$, respectively $[1,2]$. Inhaled corticosteroids (ICSs) are the mainstay of asthma therapy and are often used in COPD [3,4]. However, corticosteroids have inhibitory effects on innate and acquired immune function, and oral corticosteroids increase the risk of systemic infection. ICSs are delivered directly to the lungs and therefore ICSs might increase susceptibility to local (lung) infections. Indeed, ICS use is associated with an increased risk of pneumonia in persons with COPD and asthma [5-11]. ICS use also increases the risk of tuberculosis (TB), in both high- and low-burden regions $[12,13]$. However, little has been published examining the potential risk of nontuberculous mycobacterial pulmonary disease (NTM-PD) infections associated with ICS use.

NTM-PD significantly impairs quality of life and is often difficult to treat $[14,15]$. The pathogenesis of NTM-PD remains incompletely understood, but COPD and asthma are risk factors [16-18]. It is unclear whether the association between OLD and NTM-PD is due to structural and functional abnormalities of the diseased lung, medications used to treat airways disease, both factors combined or other factors. Only one prior study examined whether ICS use increases the risk of NTM-PD [16]. This case-control study examined the risk of NTM-PD in persons with chronic respiratory disease, dividing them into ICS nonusers, ICS ever-users and ICS current users, compared with matched population controls. The results demonstrated an association (3.8-fold increased risk in persons with COPD); however, the numbers were small (only 112 NTM-PD cases) and the authors did not control for OLD severity. The primary objective of the present study was to determine if ICS use is associated with an increased risk of NTM-PD in persons with OLD. Secondary objectives included characterising this risk by type and dose of ICS, type of OLD, and determining whether ICS use is associated with an increased risk of TB.

\section{Methods}

Study population and setting

We conducted a population-based nested case-control study using linked laboratory and health administrative databases. All registered residents of Ontario entered the study cohort on or after January 1, 2001 , if they were aged $\geqslant 66$ years, had OLD (asthma, COPD or asthma-COPD overlap syndrome (ACOS)) and filled one or more prescription(s) for OLD (any inhaled $\beta$-agonist, inhaled anticholinergic, ICS, oral corticosteroid or methylxanthine) during the study period. Cohort patients were followed until the earliest of emigration, death, loss to follow-up (no medical encounters or filled prescriptions within 12 months) or December 31, 2013.

Ontario is Canada's most populous province, with 13.5 million residents in 2013, and has a single-payer healthcare system providing universal access to medically necessary inpatient and outpatient services, and prescription drugs to adults aged $\geqslant 65$ years. Ethics approvals were obtained from the appropriate institutions.

\section{Data sources}

Health administrative data at the Institute for Clinical Evaluative Sciences (Toronto, ON, Canada) were used to identify cohort patients with OLD, demographics [19], vital status, comorbidities [20-24] and drug exposure (see supplementary material for details). Asthma and COPD were identified using validated algorithms $[25,26]$, and ACOS was defined when a patient met criteria for both asthma and COPD (not previously validated).

Mycobacterial infections were identified with Public Health Ontario's (Toronto, ON, Canada) laboratory information system, which captures $~ 95 \%$ of NTM isolates and $100 \%$ of TB isolates in the province [27]. See supplementary material for mycobacterial culture methods.

\section{Identification of cases and controls}

Cases of NTM-PD were defined using microbiological criteria: two or more sputa culture positive for the same NTM species within 2 years, or one bronchoscopy or lung biopsy or pleural specimen culture positive (positive predictive value 69.5-90\%) [28-32]. “Treated NTM-PD” was defined as NTM-PD with prescription of two or more antibiotics (see supplementary material) used to treat NTM-PD for $>90$ consecutive days in the year following laboratory confirmation of NTM. Cases of TB were defined as any culture positive for Mycobacterium tuberculosis complex organisms. We restricted NTM cases to pulmonary NTM, but allowed TB involving any organ, assuming that latent TB could theoretically be reactivated by ICS use, but extrapulmonary NTM would be very unlikely to result from ICS use. Patients with NTM isolated after January 1, 1998 (earliest date available), but prior to cohort entry $(\mathrm{n}=479)$ were not considered NTM cases, and patients with TB isolated after January 1, 1998, but within 18 months 
before cohort entry $(n=323)$ were not considered TB cases, in order to restrict our analyses to incident mycobacterial cases.

The index date for cases was defined as 8 weeks prior to identification of the first positive mycobacterial culture in the laboratory, to allow time for specimen collection, processing in the laboratory, and growth and identification of the organism. Each case was matched to up to four controls from the study cohort on index date according to age ( \pm 1 year), sex, year of cohort entry, type of OLD (asthma, COPD or ACOS) and duration of OLD ( \pm 2 years). Controls were sampled with replacement and were assigned the index date of their corresponding case patient.

\section{Drug exposure definitions}

The primary exposure was the use of ICS, described as a prescription filled for any of orally inhaled beclomethasone, budesonide, ciclesonide, fluticasone propionate or momethasone, either alone or in combination, and defined as current (end date of last filled ICS prescription $\leqslant 30$ days prior to index date), prior (end date of last filled ICS prescription 31-365 days prior to index date) and no use (never use or end date of last filled ICS prescription $>365$ days prior to index date).

\section{Statistical analysis}

We estimated odds ratios comparing ICS current and prior use with nonuse among NTM-PD cases and controls using conditional logistic regression and adjusted for covariates potentially associated with NTM-PD $[15,17,33]$, and surrogates of OLD severity and exacerbations (use of OLD medications within 1 year prior to index date, time since last hospitalisation for OLD, spirometry measured within 5 years prior to index date and use of home oxygen). The same analyses were performed among TB cases and controls, with the addition of prior NTM-PD as a covariate. See supplementary material for list of covariates included in adjusted analyses.

Pre-planned secondary analyses included stratification of exposure by type of ICS among current users, a dose-response analysis using 1-year beclomethasone equivalent [3] cumulative ICS exposure prior to the index date divided into tertiles (low, moderate and high), a subgroup analysis based on type of OLD, analyses restricted to treated NTM-PD cases and their respective controls, and sensitivity analyses with lag times of 2, 4 and 12 months prior to the index date, during which time exposures to ICSs were excluded, in an attempt to detect possible protopathic bias. We also examined the distribution of NTM species causing NTM-PD in this cohort and whether association with ICS use varied by species, and we calculated the number of patients needed to be treated with ICSs for one additional patient to be harmed (NNTH; see supplementary material for calculation) [34]. All analyses were performed using SAS Enterprise Guide version 6.2 (SAS Institute, Cary, NC, USA). All tests were two-tailed with the type I error $(\alpha)$ rate set at $5 \%$.

\section{Results}

There were 574271 Ontarians aged $\geqslant 66$ years with OLD during the study period (figure 1 ). A total of 156777 were deemed ineligible and therefore excluded, leaving 417494 patients with treated OLD in the cohort. Within this cohort, we identified 2966 cases of NTM-PD, who were matched to 11851 controls. NTM-PD patients (cases) were less likely to live in a rural setting, or to have diabetes or chronic kidney disease, and more likely to have bronchiectasis, interstitial lung disease or prior TB than control patients (table 1). NTM-PD patients also had a higher overall comorbidity score, and were more likely than controls to have been hospitalised recently for OLD, undergone spirometry, used home oxygen, used OLD medications and used immunosuppressant medications (table 1).

Within the same study cohort, we identified 327 cases of TB, who were matched to 1308 controls. Baseline characteristics for TB cases and the matched controls are provided in the supplementary material.

Current ICS use was associated with a significantly increased risk of NTM-PD compared with nonuse (crude OR (cOR) 2.87, 95\% CI 2.59-3.19; adjusted OR (aOR) 1.86, 95\% CI 1.60-2.15) (table 2); NNTH 1775 year $^{-1}$. Prior ICS use was significantly associated with NTM-PD in the unadjusted analysis, but not after adjustment.

The association between NTM-PD and ICS current use was statistically significant for fluticasone (aOR 2.09, 95\% CI 1.80-2.43), but not for budesonide (aOR 1.19, 95\% CI 0.97-1.45) or “other" ICSs (aOR 1.29, 95\% CI 0.86-1.93) (table 2). To explore whether there was a differential risk between fluticasone and budesonide, we ran two post hoc adjusted analyses. 1) We changed the reference group to current budesonide users and found that current fluticasone use was associated with a significantly increased risk of NTM-PD compared with current budesonide use (aOR 1.76, 95\% CI 1.50-2.07; p<0.001). 2) We divided current users of fluticasone and budesonide into those exposed to low, moderate or high mean 


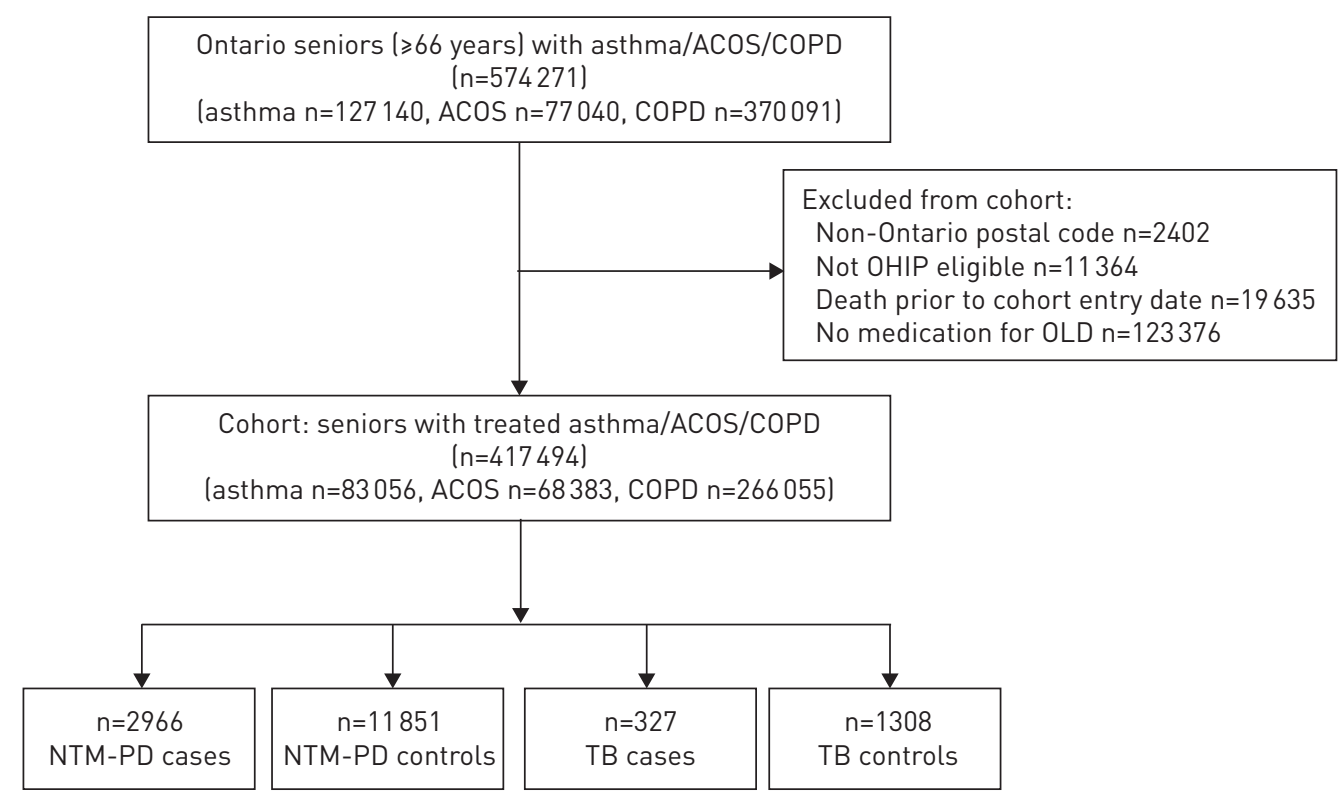

FIGURE 1 Study population. ACOS: asthma-COPD overlap syndrome; COPD: chronic obstructive pulmonary disease; OHIP: Ontario Health Insurance Plan; OLD: obstructive lung disease; NTM-PD: nontuberculous mycobacterial pulmonary disease; TB: tuberculosis.

daily dose (based on beclomethasone equivalents) [3], in order to account for the higher potency of fluticasone. With high-dose budesonide users as the reference group, we found that current use of low-, moderate- or high-dose fluticasone was associated with increased risk of NTM-PD: low-dose fluticasone aOR 1.74 (95\% CI 1.26-2.42; p<0.001), moderate-dose fluticasone aOR 1.82 (95\% CI 1.35-2.45; $\mathrm{p}<0.001$ ) and high-dose fluticasone aOR 1.89 (95\% CI 1.40-2.53; p<0.001).

A concordant dose-response relationship was evident between incident NTM-PD and cumulative ICS exposure over 1 year (table 2 and figure 2).

The association between NTM-PD and current ICS use was statistically significant for patients with COPD only (asthma absent, aOR 1.96, 95\% CI 1.62-2.36) and for patients with ACOS (aOR 1.74, 95\% CI 1.322.28), but was not statistically significant for patients with asthma only (COPD absent, aOR 1.56, 95\% CI 0.93-2.62) (table 2). However, an interaction analysis did not detect a statistically significant difference in risk between individuals with asthma, COPD or ACOS $(p=0.21)$. Therefore, the association between ICS use and NTM-PD in the asthma-only subgroup may be nonsignificant due to low power.

The association between current ICS use and NTM-PD was statistically significant when we studied only cases of NTM-PD who received antimicrobial treatment in the year following the index date (aOR 1.65, 95\% CI 1.07-2.56) (table 3).

The species causing NTM-PD in our cohort were primarily MAC (63.3\% of all NTM-PD cases) and Mycobacterium xenopi (27.3\%) (table 4). The association with ICS use did not differ significantly between these two species ( $\mathrm{p}$-value for $\mathrm{z}$-test comparing the aORs=0.61).

Current ICS use was associated with a significantly increased risk of TB in the crude analysis (cOR 1.47, 95\% CI 1.10-1.96), but not in the adjusted analysis (aOR 1.43, 95\% CI 0.95-2.16) (table 5).

Introducing lag times of 2, 4 and 12 months attenuated the magnitude of the association between current ICS use and NTM-PD slightly, but statistically significant associations persisted (aOR with 2-month lag time 1.58, 95\% CI 1.37-1.81; aOR with 4-month lag time 1.57, 95\% CI 1.37-1.80; aOR with 12-month lag time $1.68,95 \%$ CI $1.48-1.90)$.

\section{Discussion}

In our population-based study of adults aged $\geqslant 66$ years with treated OLD, current ICS use was associated with significantly increased risk of NTM-PD compared with nonuse. We observed a strong dose-response relationship between NTM-PD and cumulative ICS exposure over 1 year. The association between ICS use and NTM-PD was only found with fluticasone. Importantly, the association between ICS use and NTM-PD cases requiring antibiotic treatment implies that ICS use increases the risk of clinically significant NTM-PD. 
TABLE 1 Baseline characteristics on the index date for nontuberculous mycobacterial pulmonary disease (NTM-PD) cases and matched controls

\begin{tabular}{|c|c|c|c|}
\hline & NTM-PD cases & Controls & p-value \\
\hline Subjects & 2966 & 11851 & \\
\hline Female & $1341(45.2)$ & $5360(45.2)$ & 0.99 \\
\hline Age & $76.6 \pm 6.36$ & $76.6 \pm 6.35$ & 0.943 \\
\hline Income quintile & & & 0.893 \\
\hline 1 (lowest) & $760(25.6)$ & $2939(24.8)$ & \\
\hline 2 & $688(23.2)$ & $2617(22.1)$ & \\
\hline 3 & $523(17.6)$ & $2254(19.0)$ & \\
\hline 4 & 470 (15.8) & $2104(17.8)$ & \\
\hline 5 (highest) & $517(17.4)$ & $1868(15.8)$ & \\
\hline Missing & $8(0.3)$ & $69(0.6)$ & \\
\hline Rural residency\# & $76(2.6)$ & $1472(12.4)$ & $<0.001$ \\
\hline Suburban & $267(9.0)$ & $3188(26.9)$ & \\
\hline Urban & $2623(88.4)$ & $7191(60.7)$ & \\
\hline ADGs & $10.5 \pm 3.71$ & $9.83 \pm 3.96$ & $<0.001$ \\
\hline \multicolumn{4}{|l|}{ Comorbidities } \\
\hline Bronchiectasis & 483 (16.3) & $559(4.7)$ & $<0.001$ \\
\hline Chronic kidney disease & $297(10.0)$ & $1444(12.2)$ & 0.001 \\
\hline Diabetes mellitus & $723(24.4)$ & $3608(30.4)$ & $<0.001$ \\
\hline GORD & 704 (23.7) & $2818(23.8)$ & 0.961 \\
\hline HIV & $\leqslant 5$ & $\leqslant 5$ & 0.264 \\
\hline Interstitial lung disease & 277 (9.3) & $504(4.3)$ & $<0.001$ \\
\hline Rheumatoid arthritis & $121(4.1)$ & 412 (3.5) & 0.115 \\
\hline Prior TB & $22(0.7)$ & $8(0.1)$ & $<0.001$ \\
\hline Last COPD hospitalisation & & & $<0.001$ \\
\hline$<6$ months & $281(9.5)$ & $1143(7.7)$ & \\
\hline 6 months -5 years & $661(22.3)$ & $3131(21.1)$ & \\
\hline$>5$ years or never & 2024 (68.2) & $10543(71.2)$ & \\
\hline Last asthma hospitalisation & & & 0.017 \\
\hline$<6$ months & $25(0.8)$ & $75(0.3)$ & \\
\hline 6 months -5 years & 98 (3.3) & $461(3.1)$ & \\
\hline$>5$ years or never & 2843 (95.9) & $14281(96.4)$ & \\
\hline Spirometry within 5 years & 2369 (79.9) & $6649(56.1)$ & $<0.001$ \\
\hline Prior/current home oxygen therapy & $303(10.2)$ & $959(8.1)$ & $<0.001$ \\
\hline \multicolumn{4}{|l|}{ OLD drug exposure within 1 year } \\
\hline SABA & $1977(66.7)$ & $7314(61.7)$ & $<0.001$ \\
\hline $\mathrm{LABA}^{+}$ & $1828(61.6)$ & $4949(41.8)$ & $<0.001$ \\
\hline SAMA & $928(31.3)$ & $3132(26.4)$ & $<0.001$ \\
\hline LAMA & $1348(45.4)$ & $4006(33.8)$ & $<0.001$ \\
\hline Methylxanthine & $301(10.1)$ & $597(5.0)$ & $<0.001$ \\
\hline Oral corticosteroid & $990(33.4)$ & $2807(23.7)$ & $<0.001$ \\
\hline \multicolumn{4}{|l|}{ Other drug exposure within 1 year } \\
\hline TNF- $\alpha$ antagonist & $6(0.2)$ & $17(0.1)$ & 0.467 \\
\hline Other immunosuppressant ${ }^{\S}$ & $149(5.0)$ & $343(2.9)$ & $<0.001$ \\
\hline PPI & $1102(37.2)$ & $4335(36.6)$ & 0.561 \\
\hline
\end{tabular}

Data are presented as $n, n(\%)$ or mean \pm SD, unless otherwise stated. ADGs: aggregated diagnostic groups la general measure of comorbidity [24]); GORD: gastro-oesophageal reflux disease; TB: tuberculosis; COPD: chronic obstructive pulmonary disease; OLD: obstructive lung disease; SABA: short-acting $\beta$-agonist; LABA: long-acting $\beta$-agonist; SAMA: short-acting antimuscarinic agent; LAMA: long-acting antimuscarinic agent; TNF: tumour necrosis factor; PPI: proton pump inhibitor. ${ }^{\#}$ : based on Rural Index for Ontario, a measure of rurality designed for Ontario [19]. " : values are suppressed to prevent the disclosure of cell sizes smaller than six in accordance with contractual obligations to our data partners. ${ }^{+}$: includes LABA in a combination inhaler with inhaled corticosteroids or alone. ${ }^{\S}$ : includes methotrexate, leflunomide, azathioprine, cyclophosphamide, sulfasalazine, hydroxychloroquine, gold, cyclosporine, penicillamine, chloroquine, chlorambucil, mycophenolate mofetil, auronafin; does not include corticosteroids.

Few studies have examined the association between ICS use and NTM-PD. A Danish case-control study included an ICS analysis that was limited to northern Denmark, with 112 cases (NTM-PD patients), comprising 54 with COPD, 10 with asthma and 48 without OLD. The investigators calculated odds ratios of NTM-PD according to the presence of various types of lung disease and the additional effect of ICSs [16]. 
TABLE 2 Odds ratios for nontuberculous mycobacterial pulmonary disease (NTM-PD) according to inhaled corticosteroid (ICS) exposure

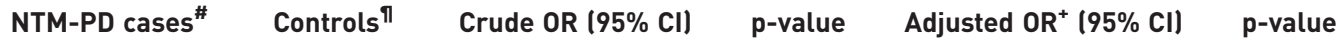

\begin{tabular}{|c|c|c|c|c|c|c|}
\hline \multicolumn{7}{|l|}{ All OLD } \\
\hline No ICS use & $592(20.0)$ & $4175(35.2)$ & 1.0 (reference) & & 1.0 (reference) & \\
\hline Prior ICS use & 469 (15.8) & $2647(22.3)$ & $1.30(1.14-1.48)$ & 0.001 & $0.94(0.80-1.11)$ & 0.465 \\
\hline Current ICS use & $1905(64.2)$ & $5029(42.4)$ & $2.87(2.59-3.19)$ & $<0.001$ & $1.86(1.60-2.15)$ & $<0.001$ \\
\hline Budesonide & $291(9.8)$ & $1070(9.0)$ & $2.05(1.75-2.40)$ & $<0.001$ & $1.19(0.97-1.45)$ & 0.089 \\
\hline Other ICS ${ }^{\Uparrow}$ & $38(1.28)$ & $180(1.5)$ & $1.59(1.11-2.28)$ & 0.012 & $1.29(0.86-1.93)$ & 0.223 \\
\hline \multicolumn{7}{|c|}{ ICS cumulative dose in 1 year } \\
\hline Moderate dose & $781(26.3)$ & $2615(22.1)$ & $2.27(2.01-2.56)$ & $<0.001$ & $1.48(1.27-1.74)$ & $<0.001$ \\
\hline High dose & $1047(35.3)$ & 2292 (19.3) & $3.58(3.17-4.03)$ & $<0.001$ & $2.28(1.94-2.68)$ & $<0.001$ \\
\hline Asthma only & 219 & 872 & & & & \\
\hline No ICS use & 81 (37.0) & $398(45.6)$ & 1.0 (reference) & & 1.0 (reference) & \\
\hline Prior ICS use & $55(25.1)$ & $236(27.1)$ & $1.18(0.80-1.74)$ & 0.406 & $1.23(0.74-2.03)$ & 0.428 \\
\hline Current ICS use & 83 (37.9) & 238 (27.3) & $1.76(1.23-2.51)$ & 0.002 & $1.56(0.93-2.62)$ & 0.095 \\
\hline COPD only & 1837 & 7346 & & & & \\
\hline No ICS use & 365 (19.9) & 2744 (37.3) & 1.0 (reference) & & 1.0 (reference) & \\
\hline Prior ICS use & 279 (15.2) & $1574(21.4)$ & $1.40(1.18-1.65)$ & $<0.001$ & $0.98(0.79-1.20)$ & 0.827 \\
\hline Current ICS use & $1193(64.9)$ & $3028(41.2)$ & $3.20(2.79-3.66)$ & $<0.001$ & $1.96(1.62-2.36)$ & $<0.001$ \\
\hline
\end{tabular}

Data are presented as $\mathrm{n}(\%)$, unless otherwise stated. OLD: obstructive lung disease; ACOS: asthma-COPD overlap syndrome; COPD: chronic obstructive pulmonary disease. \#: $n=2966$. n: $^{n}=11851 .{ }^{+}$: adjusted for income, rurality, aggregated diagnostic groups, comorbidities (bronchiectasis, chronic kidney disease, gastro-oesophageal reflux disease, HIV, interstitial lung disease, rheumatoid arthritis), prior tuberculosis, medication use limmunosuppressants lincludes methotrexate, leflunomide, azathioprine, cyclophosphamide, sulfasalazine, hydroxychloroquine, gold, cyclosporine, penicillamine, chloroquine, chlorambucil, mycophenolate mofetil, auronafin; does not include corticosteroids), proton pump inhibitors), and surrogates of severity of OLD and exacerbations of OLD (medications for OLD (any inhaled $\beta$-agonist, inhaled anticholinergic, oral corticosteroid or methylxanthine), hospitalisation for OLD, spirometry, home oxygen use).

Direct comparison with our results is difficult due to methodological differences, but some similarities are apparent. They found that compared with the absence of COPD, the presence of COPD without ICS use had an associated aOR for NTM-PD of 7.6 (95\% CI 3.4-16.8), while the presence of COPD with current ICS use had an associated aOR for NTM-PD of 29.1 (95\% CI 13.3-63.8). The inclusion of current ICS use increased the aOR estimate 3.8-fold. Like us, they also found a dose-response relationship, a greater association with fluticasone than budesonide and no significant association in a subgroup of asthma patients. The major difference between our findings and theirs relates to the magnitude of the risk estimates, which appear greater with their study (in the COPD subgroup, aOR 3.8 in

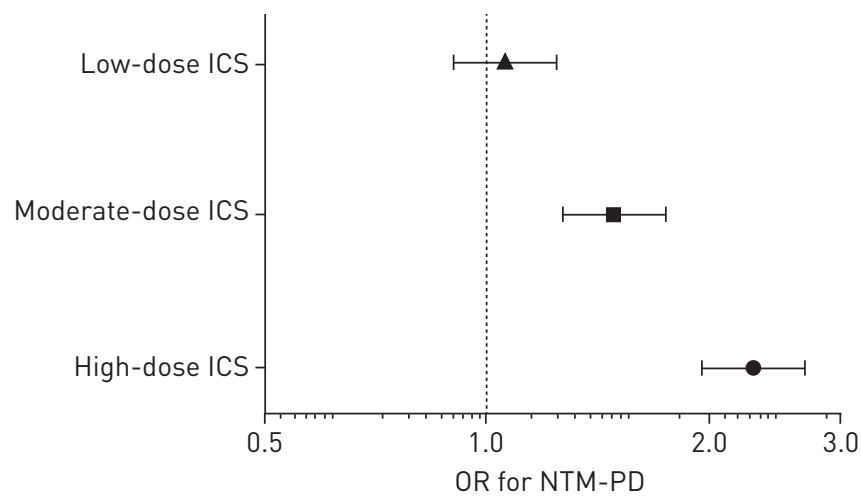

FIGURE 2 Odds ratios ( $95 \% \mathrm{Cls}$ ) for nontuberculous mycobacterial pulmonary disease (NTM-PD) by tertiles of cumulative inhaled corticosteroid (ICS) dose in the year prior to index date, compared with no ICS use. 


\begin{tabular}{|c|c|c|c|c|c|c|}
\hline & Treated NTM-PD cases ${ }^{\pi}$ & Controls $^{+}$ & Crude OR $(95 \% \mathrm{CI})$ & p-value & Adjusted OR $(95 \% \mathrm{CI})$ & $\mathrm{p}$-value \\
\hline No ICS use & 77 (19.4) & $504(31.7)$ & 1.00 (reference) & & 1.00 (reference) & \\
\hline Prior ICS use & 65 (16.3) & $353(22.2)$ & $1.25(0.87-1.79)$ & 0.234 & $1.13(0.72-1.78)$ & 0.605 \\
\hline Current ICS use & $256(64.3)$ & 735 (46.2) & $2.39(1.79-3.19)$ & $<0.001$ & $1.65(1.07-2.56)$ & 0.024 \\
\hline
\end{tabular}

Data are presented as $\mathrm{n}(\%)$, unless otherwise stated. ${ }^{\#}$ : defined as persons who met criteria for NTM-PD and were also prescribed at least two antibiotics used to treat NTM-PD (see supplementary material for list of antibiotics) for $>90$ days in the year following laboratory confirmation of NTM. ๆ: $n=398 .{ }^{+}: n=1592 .^{\S}$ : adjusted for income, rurality, aggregated diagnostic groups, comorbidities (bronchiectasis, chronic kidney disease, gastro-oesophageal reflux disease, HIV, interstitial lung disease, rheumatoid arthritis), prior tuberculosis, medication use (immunosuppressants lincludes methotrexate, leflunomide, azathioprine, cyclophosphamide, sulfasalazine, hydroxychloroquine, gold, cyclosporine, penicillamine, chloroquine, chlorambucil, mycophenolate mofetil, auronafin; does not include corticosteroids), proton pump inhibitors) and surrogates of severity of obstructive lung disease (OLD) (medications for OLD lany inhaled $\beta$-agonist, inhaled anticholinergic, oral corticosteroid or methylxanthine), hospitalisation for OLD, spirometry, home oxygen use).

their study versus aOR 1.96 (95\% CI 1.62-2.36) in ours). This discrepancy may be due to differing definitions of NTM-PD (they required three sputa culture positive, rather than two), differing definitions of current and no ICS exposure (they defined current exposure as any exposure in the preceding 6 months and nonuse as never used), and differences in covariates (we included markers of OLD severity/ exacerbations in the multivariable analysis, which may have attenuated the risk). Nonetheless, our much larger study confirms their findings and our inclusion of markers of OLD severity/exacerbations suggests that this association is unlikely confounded by severity of OLD. Additionally, our finding of a significant association between ICS use and antibiotic-treated NTM-PD suggests that ICS use increases the risk of clinically important NTM-PD.

Hojo et al. [35] performed a single-institution nested case-control study in patients with asthma and found that cases of NTM-PD received a higher daily dose of ICS $(650 \pm 257 \mu \mathrm{g}$ of fluticasone propionate equivalent) than controls $(358 \pm 273 \mu \mathrm{g})(\mathrm{p}<0.01)$ for a longer duration $(8.2 \pm 4.8$ versus $5.3 \pm 4.9$ years; $\mathrm{p}<0.01)$. These findings are consistent with our finding of a strong dose-response relationship between cumulative ICS exposure over 1 year and NTM-PD.

We did not find a significant association between ICS use and TB. In contrast, a similar nested casecontrol study using health administrative data in Quebec, Canada, found a significant association between current ICS use (30 days prior to index) and TB (relative risk 1.33, 95\% CI 1.04-1.71) [13]. Our risk estimate (aOR 1.43, 95\% CI 0.95-2.16) is similar to theirs; however, with fewer TB cases (327 versus 564) we suspect we lacked power to detect a significant association. Another study, based on data from one hospital in South Korea, employed a cohort design and found significant associations between ICS use and $\mathrm{TB}$ in patients with a normal chest radiograph (hazard ratio 9.1, 95\% CI 1.21-81) and in patients with radiological features of old TB (hazard ratio $24.9,95 \%$ CI 3.1-201) [12].

TABLE 4 Distribution of nontuberculous mycobacterial species causing pulmonary disease (NTM-PD) and odds ratios comparing current inhaled corticosteroid (ICS) use with no ICS use

\begin{tabular}{lcccr} 
& NTM-PD cases $^{\#}$ & Controls $^{\text {I }}$ & Adjusted OR $^{+}(95 \%$ CI) & p-value \\
\hline MAC & $1877(63.3)$ & 7495 & $1.87(1.55-2.55)$ & $<0.001$ \\
Mycobacterium xenopi & $811(27.3)$ & 3244 & $2.04(1.54-2.70)$ & $<0.001$ \\
Other NTM & $278(9.4)$ & 1112 & $1.55(0.95-2.52)$ & 0.077 \\
\hline
\end{tabular}

Data are presented as $\mathrm{n}(\%)$, unless otherwise stated. MAC: Mycobacterium avium complex comprised of Mycobacterium avium and Mycobacterium intracellulare. ${ }^{\#}: \mathrm{n}=2966 .{ }^{\text {ๆ: }} \mathrm{n}=11851$. $^{+}$: adjusted odds ratio comparing current ICS use with no ICS use; adjusted for income, rurality, aggregated diagnostic groups, comorbidities (bronchiectasis, chronic kidney disease, gastro-oesophageal reflux disease, HIV, interstitial lung disease, rheumatoid arthritis), prior tuberculosis, medication use (immunosuppressants (includes methotrexate, leflunomide, azathioprine, cyclophosphamide, sulfasalazine, hydroxychloroquine, gold, cyclosporine, penicillamine, chloroquine, chlorambucil, mycophenolate mofetil, auronafin; does not include corticosteroids), proton pump inhibitors), and surrogates of severity of OLD and exacerbations of obstructive lung disease (OLD) (medications for OLD lany inhaled $\beta$-agonist, inhaled anticholinergic, oral corticosteroid or methylxanthine), hospitalisation for OLD, spirometry, home oxygen use). 
TABLE 5 Odds ratios for tuberculosis (TB) according to inhaled corticosteroid (ICS) exposure

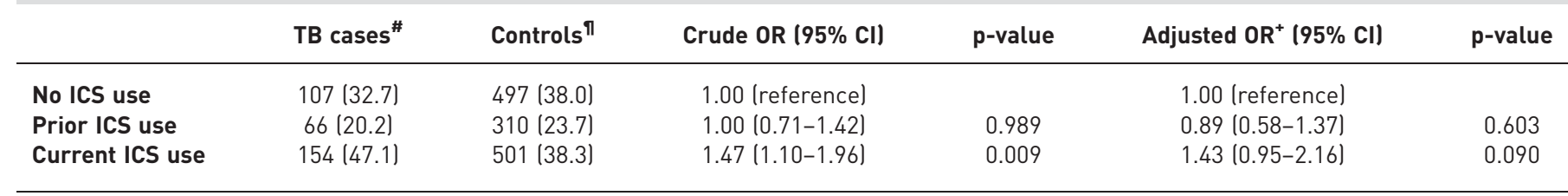

Data are presented as $n(\%)$, unless otherwise stated. ${ }^{\#}: n=327 .{ }^{~}$ : $n=1308 .{ }^{+}$: adjusted for income, rurality, aggregated diagnostic groups, comorbidities (bronchiectasis, chronic kidney disease, gastro-oesophageal reflux disease, HIV, interstitial lung disease, rheumatoid arthritis), prior tuberculosis, prior nontuberculous mycobacterial pulmonary disease, medication use limmunosuppressants (includes methotrexate, leflunomide, azathioprine, cyclophosphamide, sulfasalazine, hydroxychloroquine, gold, cyclosporine, penicillamine, chloroquine, chlorambucil, mycophenolate mofetil, auronafin; does not include corticosteroids), proton pump inhibitors), and surrogates of severity of obstructive lung disease (OLD) (medications for OLD lany inhaled $\beta$-agonist, inhaled anticholinergic, oral corticosteroid, or methylxanthine), hospitalisation for OLD, spirometry, home oxygen use).

Multiple prior studies have demonstrated an increased risk of pneumonia associated with ICS use in persons with COPD [5-10]. Fluticasone is associated with a greater risk of pneumonia than budesonide, although data are conflicting regarding whether this difference remains significant when examining only serious pneumonias $[7,10]$. Similar to the pneumonia data, we found that fluticasone had a significantly greater risk of NTM-PD than budesonide, which did not pose a significant risk of NTM-PD after adjustment. The differential risk of lung infection posed by fluticasone and budesonide is likely due to differences in pharmacokinetic and pharmacodynamic properties [7]. Fluticasone has a greater effect on glucocorticoid receptors, is more lipophilic and has a longer half-life than budesonide [36]. These properties increase fluticasone's pulmonary anti-inflammatory effects relative to budesonide, but may also increase the potential for pulmonary immunosuppression.

The significant association between ICS and NTM-PD after adjustment for severity of OLD, the differential risk of NTM-PD posed by fluticasone and budesonide (and consistency with pneumonia studies), and the strong dose-response relationship between cumulative ICS exposure and incident NTM-PD all suggest a causal relationship between ICS and NTM-PD. ICS is likely one casual factor in a multifactorial pathway that leads to NTM-PD in patients with OLD, with structural lung damage (emphysema and bronchiectasis) and decreased mucociliary clearance likely also contributing. There is scientific rationale for a causal relationship between ICSs and lung infection; inhaled fluticasone impairs pulmonary macrophage cytokine production and nitric oxide synthesis, and impedes intracellular pathogen control in mice [37]. Additionally, ICSs reduce frequencies of mucosal-associated invariant T (MAIT)-cells in blood and bronchial tissue of patients with COPD [38]. MAIT-cells contribute to protection against mycobacterial infections [39,40], and are numerically and functionally deficient in patients with NTM [41].

Strengths of our study include the large number of NTM-PD cases and the adjustment for multiple possible confounders, including surrogates for OLD severity. One limitation is that we only included people aged $\geqslant 66$ years. Additionally, we did not have data on lung function (e.g. forced expiratory volume in $1 \mathrm{~s}$ ) or the extent of emphysema, so residual confounding by severity of lung disease may persist. Also, we defined NTM-PD and TB cases microbiologically. Microbiological-based outcome definitions have $100 \%$ positive predictive value for TB, but imperfect sensitivity (55-79\%) [32]. Our microbiological-based NTM-PD definition, based upon the American Thoracic Society/Infectious Diseases Society of America microbiological criteria for NTM-PD, has shown good positive predictive value $(69.5-90 \%$ for all NTM species ( \pm Mycobacterium gordonae) [28-31] and $89.8 \%$ for MAC [42], the majority of our cases), but likely misclassified some patients with NTM colonisation as having disease, potentially impacting the validity of risk estimates.

Our findings contribute to the body of evidence informing clinicians on the safety of ICSs. We found that ICS use increases the risk of NTM-PD; however, NTM-PD is still relatively rare. Our NNTH for NTM-PD of 1775 year $^{-1}$ is much higher than the NNTH of 20-33 year ${ }^{-1}$ for pneumonia in COPD patients [43]. However, our NNTH includes all adults aged $\geqslant 66$ years with treated OLD and ICSs of any formulation or dose. The NNTH may be significantly lower in COPD patients prescribed high-dose fluticasone. Therefore, we believe the risk of NTM-PD should be considered when prescribing ICSs, especially since NTM-PD is a chronic infection that is extremely difficult to treat. We think this is particularly relevant for patients with COPD who may have other risk factors for NTM-PD (i.e. bronchiectasis, emphysema or prior sputum positive for NTM) and/or in whom the potential benefits of ICS are unclear (e.g. a patient with infrequent exacerbations). 
To conclude, in persons with OLD, ICS use is associated with an increased risk of NTM-PD. Clinicians should consider this risk when prescribing ICSs to patients with OLD and if they are needed, strive to use the lowest effective dose. Future research comparing differential risks associated with different ICS types may be helpful.

\section{Acknowledgements}

Author contributions: All authors contributed substantially to the conception and design of this work. A. MarchandAustin performed the data acquisition. M.A. Campitelli and H. Lu performed the analysis. S.K. Brode drafted the article. All authors revised the article critically for important intellectual content and gave final approval of the version to be published. The sponsors had no role in the development of the research or the manuscript.

\section{References}

1 Gershon AS, Warner L, Cascagnette P, et al. Lifetime risk of developing chronic obstructive pulmonary disease: a longitudinal population study. Lancet 2011; 378: 991-996.

2 To T, Wang C, Guan J, et al. What is the lifetime risk of physician-diagnosed asthma in Ontario, Canada? Am J Respir Crit Care Med 2010; 181: 337-343.

3 Global Initiative for Asthma. Global Strategy for Asthma Management and Prevention. 2016. www.ginasthma.org Date last accessed: June 26, 2016.

4 Van Andel AE, Reisner C, Menjoge SS, et al. Analysis of inhaled corticosteroid and oral theophylline use among patients with stable COPD from 1987 to 1995 . Chest 1999; 115: 703-707.

5 Singh S, Amin AV, Loke YK. Long-term use of inhaled corticosteroids and the risk of pneumonia in chronic obstructive pulmonary disease: a meta-analysis. Arch Intern Med 2009; 169: 219-229.

6 Drummond MB, Dasenbrook EC, Pitz MW, et al. Inhaled corticosteroids in patients with stable chronic obstructive pulmonary disease: a systematic review and meta-analysis. JAMA 2008; 300: 2407-2416.

7 Suissa S, Patenaude V, Lapi F, et al. Inhaled corticosteroids in COPD and the risk of serious pneumonia. Thorax 2013; 68: 1029-1036.

8 Crim C, Calverley PM, Anderson JA, et al. Pneumonia risk in COPD patients receiving inhaled corticosteroids alone or in combination: TORCH study results. Eur Respir J 2009; 34: 641-647.

9 Janson C, Larsson K, Lisspers KH, et al. Pneumonia and pneumonia related mortality in patients with COPD treated with fixed combinations of inhaled corticosteroid and long acting $\beta_{2}$ agonist: observational matched cohort study (PATHOS). BMJ 2013; 346: f3306.

10 Kew KM, Seniukovich A. Inhaled steroids and risk of pneumonia for chronic obstructive pulmonary disease. Cochrane Database Syst Rev 2014; 3: CD010115.

11 McKeever T, Harrison TW, Hubbard R, et al. Inhaled corticosteroids and the risk of pneumonia in people with asthma: a case-control study. Chest 2013; 144: 1788-1794.

12 Kim JH, Park JS, Kim KH, et al. Inhaled corticosteroid is associated with an increased risk of TB in patients with COPD. Chest 2013; 143: 1018-1024.

13 Brassard P, Suissa S, Kezouh A, et al. Inhaled corticosteroids and risk of tuberculosis in patients with respiratory diseases. Am J Respir Crit Care Med 2011; 183: 675-678.

14 Mehta M, Marras TK. Impaired health-related quality of life in pulmonary nontuberculous mycobacterial disease. Respir Med 2011; 105: 1718-1725.

15 Griffith DE, Aksamit T, Brown-Elliott BA, et al. An official ATS/IDSA statement: diagnosis, treatment, and prevention of nontuberculous mycobacterial diseases. Am J Respir Crit Care Med 2007; 175: 367-416.

16 Andrejak C, Nielsen R, Thomsen VO, et al. Chronic respiratory disease, inhaled corticosteroids and risk of non-tuberculous mycobacteriosis. Thorax 2013; 68: 256-262.

17 Dirac MA, Horan KL, Doody DR, et al. Environment or host? A case-control study of risk factors for Mycobacterium avium complex lung disease. Am J Respir Crit Care Med 2012; 186: 684-691.

18 Marras TK, Campitelli MA, Kwong JC, et al. Risk of nontuberculous mycobacterial pulmonary disease with obstructive lung disease. Eur Respir J 2016; 48: 928-931.

19 Kralj B. Measuring "rurality" for purposes of health-care planning: an empirical measure for Ontario. Ontario Med Rev 2000; 10: 33-52.

20 Hux JE, Ivis F, Flintoft V, et al. Diabetes in Ontario: determination of prevalence and incidence using a validated administrative data algorithm. Diabetes Care 2002; 25: 512-516.

21 Lopushinsky SR, Covarrubia KA, Rabeneck L, et al. Accuracy of administrative health data for the diagnosis of upper gastrointestinal diseases. Surg Endosc 2007; 21: 1733-1737.

22 Vlasschaert MEO, Bejaimal SAD, Hackam DG, et al. Validity of administrative database coding for kidney disease: a systematic review. Am J Kidney Dis 2011; 57: 29-43.

23 Widdifield J, Bombardier C, Bernatsky S, et al. An administrative data validation study of the accuracy of algorithms for identifying rheumatoid arthritis: the influence of the reference standard on algorithm performance. BMC Musculoskelet Disord 2014; 15: 216.

24 Reid RJ, MacWilliam L, Verhulst L, et al. Performance of the ACG case-mix system in two Canadian provinces. Med Care 2001; 39: 86-99.

25 Gershon AS, Wang C, Guan J, et al. Identifying patients with physician-diagnosed asthma in health administrative databases. Can Respir J 2009; 16: 183-188.

26 Gershon AS, Wang C, Guan J, et al. Identifying individuals with physician diagnosed COPD in health administrative databases. COPD 2009; 6: 388-394.

27 Al Houqani M, Jamieson F, Cheodore P, et al. Isolation prevalence of pulmonary nontuberculous mycobacteria in Ontario in 2007. Can Respir J 2011; 18: 19-24.

28 Andrejak C, Thomsen VO, Johansen IS, et al. Nontuberculous pulmonary mycobacteriosis in Denmark: incidence and prognostic factors. Am J Respir Crit Care Med 2010; 181: 514-521.

29 Prevots DR, Shaw PA, Strickland D, et al. Nontuberculous mycobacterial lung disease prevalence at four integrated health care delivery systems. Am J Respir Crit Care Med 2010; 182: 970-976. 
30 Marras TK, Mehta M, Chedore P, et al. Nontuberculous mycobacterial lung infections in Ontario, Canada: clinical and microbiological characteristics. Lung 2010; 188: 289-299.

31 Winthrop KL, McNelley E, Kendall B, et al. Pulmonary nontuberculous mycobacterial disease prevalence and clinical features: an emerging public health disease. Am J Respir Crit Care Med 2010; 182: 977-982.

32 Winthrop KL, Baxter R, Liu L, et al. The reliability of diagnostic coding and laboratory data to identify tuberculosis and nontuberculous mycobacterial disease among rheumatoid arthritis patients using anti-tumor necrosis factor therapy. Pharmacoepidemiol Drug Saf 2011; 20: 229-235.

33 Brode SK, Jamieson FB, Ng R, et al. Increased risk of mycobacterial infections associated with anti-rheumatic medications. Thorax 2015; 70: 677-682.

34 Bjerre LM, LeLorier J. Expressing the magnitude of adverse effects in case-control studies: "the number of patients needed to be treated for one additional patient to be harmed". BMJ 2000; 320: 503-506.

35 Hojo M, Iikura M, Hirano S, et al. Increased risk of nontuberculous mycobacterial infection in asthmatic patients using long-term inhaled corticosteroid therapy. Respirology 2012; 17: 185-190.

36 Derendorf H, Nave R, Drollmann A, et al. Relevance of pharmacokinetics and pharmacodynamics of inhaled corticosteroids to asthma. Eur Respir J 2006; 28: 1042-1050.

37 Patterson CM, Morrison RL, D’Souza A, et al. Inhaled fluticasone propionate impairs pulmonary clearance of Klebsiella pneumoniae in mice. Respir Res 2012; 13: 40.

38 Hinks TS, Wallington JC, Williams AP, et al. Steroid-induced deficiency of mucosal-associated invariant T cells in the chronic obstructive pulmonary disease lung. implications for nontypeable Haemophilus influenzae infection. Am J Respir Crit Care Med 2016; 194: 1208-1218.

39 Chua WJ, Truscott SM, Eickhoff CS, et al. Polyclonal mucosa-associated invariant T cells have unique innate functions in bacterial infection. Infect Immun 2012; 80: 3256-3267.

40 Le Bourhis L, Martin E, Peguillet I, et al. Antimicrobial activity of mucosal-associated invariant T cells. Nat Immunol 2010; 11: 701-708.

41 Kwon YS, Cho YN, Kim MJ, et al. Mucosal-associated invariant T cells are numerically and functionally deficient in patients with mycobacterial infection and reflect disease activity. Tuberculosis 2015; 95: 267-274.

42 Jankovic M, Sabol I, Zmak L, et al. Microbiological criteria in non-tuberculous mycobacteria pulmonary disease: a tool for diagnosis and epidemiology. Int J Tuberc Lung Dis 2016; 20: 934-940.

43 Suissa S. Number needed to treat in COPD: exacerbations versus pneumonias. Thorax 2013; 68: 540-543. 